\title{
Covalent IR820-PEG-diamine nanoconjugates for theranostic applications in cancer
}

\author{
This article was published in the following Dove Press journal: \\ International Journal of Nanomedicine \\ 6 October 2014 \\ Number of times this article has been viewed
}

\author{
Alicia Fernandez- \\ Fernandez ${ }^{1,2}$ \\ Romila Manchanda ${ }^{1,3}$ \\ Denny A Carvajal ${ }^{1,4}$ \\ Tingjun Lei ${ }^{1,5}$ \\ Supriya Srinivasan' \\ Anthony J McGoron'
}

'Biomedical Engineering Department, Florida International University, Miami, FL, USA; ${ }^{2}$ Physical Therapy Department, Nova Southeastern University, Fort Lauderdale, FL, USA; ${ }^{3}$ Chemistry Department, Galgotias University, Greater Noida, UP, India; ${ }^{4}$ Mount Sinai Medical Center,

${ }^{5}$ Cirle, Miami, FL, USA
Correspondence: Anthony J McGoron Biomedical Engineering Department, Florida International University, 10555 W Flagler St, EC 26I4, Miami, FL, 33174, USA

$\mathrm{Tel}+\mid 305348$ I352

Fax +I 3053486954

Email mcgorona@fiu.edu
Abstract: Near-infrared dyes can be used as theranostic agents in cancer management, based on their optical imaging and localized hyperthermia capabilities. However, their clinical translatability is limited by issues such as photobleaching, short circulation times, and nonspecific biodistribution. Nanoconjugate formulations of cyanine dyes, such as IR820, may be able to overcome some of these limitations. We covalently conjugated IR 820 with $6 \mathrm{kDa}$ polyethylene glycol (PEG)-diamine to create a nanoconjugate (IRPDcov) with potential for in vivo applications. The conjugation process resulted in nearly spherical, uniformly distributed nanoparticles of approximately $150 \mathrm{~nm}$ diameter and zeta potential $-0.4 \pm 0.3 \mathrm{mV}$. The IRPDcov formulation retained the ability to fluoresce and to cause hyperthermia-mediated cell-growth inhibition, with enhanced internalization and significantly enhanced cytotoxic hyperthermia effects in cancer cells compared with free dye. Additionally, IRPDcov demonstrated a significantly longer $(P<0.05)$ plasma half-life, elimination half-life, and area under the curve (AUC) value compared with IR820, indicating larger overall exposure to the theranostic agent in mice. The IRPDcov conjugate had different organ localization than did free IR820, with potential reduced accumulation in the kidneys and significantly lower $(P<0.05)$ accumulation in the lungs. Some potential advantages of IR820-PEG-diamine nanoconjugates may include passive targeting of tumor tissue through the enhanced permeability and retention effect, prolonged circulation times resulting in increased windows for combined diagnosis and therapy, and further opportunities for functionalization, targeting, and customization. The conjugation of PEG-diamine with a near-infrared dye provides a multifunctional delivery vector whose localization can be monitored with noninvasive techniques and that may also serve for guided hyperthermia cancer treatments.

Keywords: fluorescent imaging, hyperthermia, image-guided therapy, nanotechnology

\section{Introduction}

The development of multifunctional agents for cancer management is a rapidly growing field that can result in improved diagnosis and therapy for patients with cancer. The use of multifunctional modalities that possess diagnostic and therapeutic properties, referred to as theranostics, enables a combined approach to detection and treatment. ${ }^{1-6}$ One of the main challenges in combining early diagnosis and therapy is that imaging and therapeutic probes are typically different in terms of selectivity and biodistribution. ${ }^{7}$ This complicates the process of monitoring responses to treatment, which in turn creates difficulties in planning, timing, and assessing the success of interventions. In a multifunctional agent, the ability to follow the distribution of an agent in vivo, thanks to its imaging capabilities, enables the localization of the target site, which can be followed by the activation of the therapeutic modality once the agent has reached its desired destination. This ensures that therapy occurs at the desired site and at the appropriate time. Multifunctional formulations can also allow real-time monitoring of the effect of therapy. By combining both therapy and diagnostic capabilities into 
a single platform, theranostic agents provide clinicians with a multipurpose tool that can be used to detect, image, treat, and monitor therapeutic response over time. ${ }^{8}$ As a result, clinicians can implement therapy at an earlier stage, as well as achieve increased safety, sensitivity, and efficacy in heterogeneously natured diseases, such as cancer., ${ }^{8,9}$

Near-infrared (NIR) dyes can be used as theranostic agents in cancer management, based on their optical imaging and localized hyperthermia capabilities; however, they have important limitations in their applicability for theranostic use, especially in terms of photobleaching, in vivo instability, and nonspecific biodistribution. ${ }^{10}$ Our group has studied the properties of the cyanine dye IR820, and we provided the first detailed report of its potential for use in theranostics. ${ }^{11}$ IR820 is structurally similar to indocyanine green (ICG), which is a clinically approved cyanine dye, but IR820 has improved in vitro and in vivo stability. Our prior work has shown that IR820 is a feasible agent in experimental models of imaging and hyperthermia, and could be an alternative to ICG when greater stability, longer image collection times, or more predictable peak locations are desirable. ${ }^{11}$ Our group has also developed and characterized several formulations for combined chemotherapy, imaging, and hyperthermia, using chemotherapy agents and NIR dyes. ${ }^{12,13}$

Several recent studies have applied IR820 dye or conjugates of the dye for in vivo applications. Prajapati et al used IR820 as a blood pool contrast agent to image tissue injuries and tumors in mice. ${ }^{14}$ Pandey et al conjugated IR820 with a photodynamic therapy drug and studied the resulting conjugate in mice, with IR820 being used exclusively for its imaging role. ${ }^{15}$ Masotti et al conjugated IR820 with polyethylenimine (PEI) for DNA binding applications and in vivo imaging. ${ }^{16}$ Thierry et $\mathrm{al}^{17}$ prepared poly(allylamine hydrochloride)-poly(acrylic acid)-coated magnetic iron oxide and gold nanoparticles, which were loaded with cisplatin as well as with a conjugate of IR820 and PEI, following the work done by Masotti et al. None of these studies exploited the inherent ability of IR820 to be used as a dual imaging and hyperthermia agent. Our group has developed several IR820-based theranostic formulations, including IR820loaded polymeric nanoparticles as well as IR820-chitosan conjugates and ionic IR820 polyethylene glycol (PEG)diamine conjugates, and we have characterized the imaging and hyperthermia capabilities of these agents. ${ }^{18-21}$

Based on its optical and hyperthermia-generation properties, IR820 is a promising theranostic agent; however, clinical translation of NIR-imaging and hyperthermia approaches in cancer must overcome the challenges presented by free-dye formulations, in terms of plasma circulation times and nonspecific biodistribution. The creation of nanoformulations of IR820 provides some opportunities to improve in vivo stability and target delivery. Nanosize therapeutic and diagnostic agents can be tailored to a specific application by manipulating their size, shape, surface characteristics, and other physiochemical properties. They are also less susceptible to reticuloendothelial system clearance and have better penetration into tissues and cells than do larger size agents, ${ }^{22,23}$ and passive targeting to tumor sites can be achieved by the enhanced permeability and retention effect (EPR). ${ }^{24}$

We chose to conjugate IR820 with PEG because the presence of PEG in nanoformulations can reduce immune interactions and result in improved plasma circulation times. ${ }^{25}$ Longer exposure to the theranostic agent provides a wider window of opportunity for diagnosis and therapy, and allows more flexibility in terms of the timing of one with respect to the other, as needed for a specific application. Additionally, PEG can be used as a linker to conjugate other moieties and impart new functionality, such as specific targeting. Covalent attachment of PEG does not affect side chain properties in solution so that formulations covalently bound to PEG will maintain their functionality. ${ }^{26,27}$

Our group previously reported the formulation of ionic IR820-PEG-diamine nanoconjugates, which showed enhanced cellular internalization in cancer cells for imaging purposes compared with the free dye, and significantly enhanced hyperthermia-mediated cytotoxicity in MES-SA and MES-SA/Dx5 (Dx5) cancer cells compared with the hyperthermia achieved using free dye. ${ }^{21}$ Although promising, the ionic nanoconjugates only improved on the stability of the free dye for up to 4 hours in a phosphate buffer. Given that IR820 retained its ability to generate heat and fluoresce when interacting in close proximity with PEG as part of an ionic formulation, we hypothesized that creating a covalent formulation could further improve on the stability of the lattice and, in turn, result in nanosize conjugates that might be used in in vivo applications with improved pharmacokinetic profiles compared with the free dye, while retaining its theranostic properties. Thus, we prepared and characterized covalent conjugates of IR820 and PEG-diamine (IRPDcov).

The size and surface morphology of these nanoconjugates were characterized by transmission electron microscopy (TEM), conjugation was confirmed by proton nuclear magnetic resonance $\left({ }^{1} \mathrm{H} \mathrm{NMR}\right)$, and the optical properties in solution were studied using spectrofluorometry and spectrophotometry. We explored possible applications through in vitro experiments with three different human cancer cell 
lines (MES-SA, Dx5, and SKOV-3) to investigate the feasibility of using the nanoconjugates as imaging agents and to determine whether this formulation retained the ability to induce hyperthermic cell killing. Finally, we performed pharmacokinetics and biodistribution studies in mice to compare IRPDcov, free IR820 and ICG, and to determine whether the nanoconjugates show an advantage with respect to free-cyanine dyes in terms of in vivo applications.

\section{Methods}

\section{Nanoconjugate preparation}

PEG-diamine (6 kDa), IR820, methanol, dimethylsulfoxide (DMSO) ( $>99.9 \%$ reagent grade), and triethylamine were purchased from Sigma-Aldrich Corp., (St Louis, MO, USA).
A mixture of PEG-diamine (6 kDa, $30 \mathrm{mg} / 5 \mathrm{~mL}$ ) and IR820 $(9.4 \mathrm{mg} / 5 \mathrm{~mL})$ in aprotic methanol was refluxed for 4 hours in the presence of distilled triethylamine (2 eq). The molar ratio was 1:2, PEG-diamine:IR820, based on available functional groups (two amines in PEG-diamine and one chloro group in IR820). The reaction scheme (Figure 1) involves covalent attachment of the amine group in PEG-diamine to the cyclohexene in IR820 at the chloro-substituted position via nucleophilic substitution, with displacement of the chlorine atom and production of triethylamine hydrochloride. After the reaction progressed to completion, the sample was concentrated, and the residue was dissolved in $5 \mathrm{~mL}$ of water, followed by dialysis against water, using a molecular weight cutoff (MWCO) $3.5 \mathrm{kDa}$, for 24 hours. The nanoconjugates<smiles></smiles>

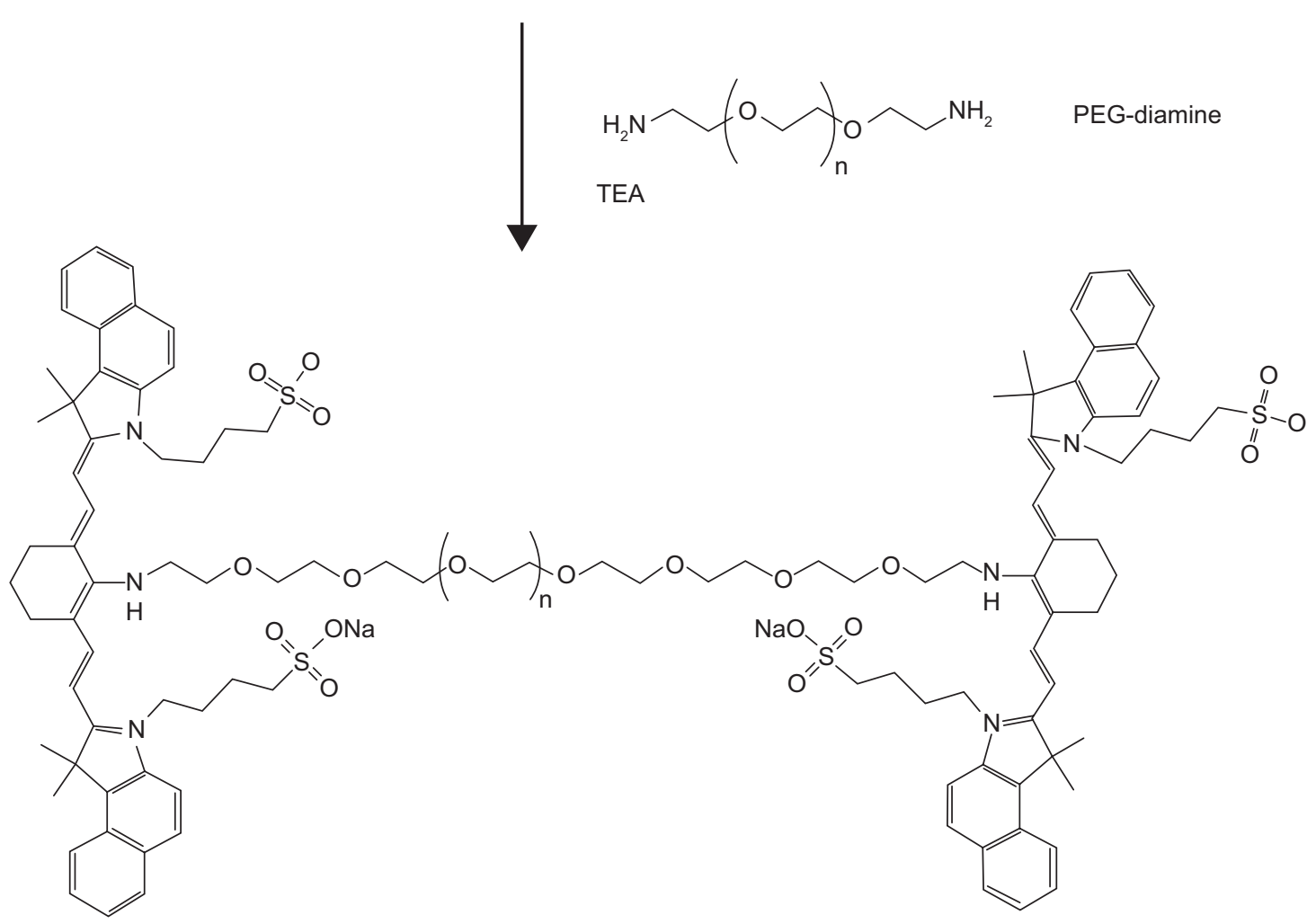

PEG-diamine-IR820

Figure I Reaction scheme and formulation of covalent IR820-PEG-diamine nanoconjugate (IRPDcov). Abbreviations: IRPDcov, covalent conjugates of IR820 and PEG-diamine; PEG, polyethylene glycol; TEA, triethylamine. 
were freeze-dried (FreeZone Plus 6 Liter Cascade Console Freeze Dry System; Labconco Corp., Kansas City, MO, USA) for 24 hours and stored in powder form at $4{ }^{\circ} \mathrm{C}$ for further use. The choice of $6 \mathrm{kDa}$ PEG-diamine was based on the criteria of resulting nanoparticle size, nanoparticle charge, reduced steric hindrance, and conservation of fluorescent and hyperthermic properties of the dye once combined with PEG-diamine.

\section{Nanoconjugate characterization}

Freeze-dried IRPDcov particles were resuspended in distilled water and later dropped onto a copper grid and dried at room temperature. The surface morphology of the samples was observed under a TEM (CM 200; FEI, Hillsboro, Oregon, USA) operated at $200-\mathrm{keV}$ pulse at different resolutions. The nanoconjugate zeta potential was measured using a Zetasizer Nano ZS (Malvern Instruments, Malvern, UK) employing a nominal $5 \mathrm{~mW}$ helium-neon ( $\mathrm{He} \mathrm{Ne}$ ) laser operating at $633 \mathrm{~nm}$ wavelength. The scattered light was detected at a $135^{\circ}$ angle. The refractive index (1.33) and the viscosity $(0.89 \mathrm{cP})$ of ultrapure water at $25^{\circ} \mathrm{C}$ were used for measurements. For ${ }^{1} \mathrm{H}$ NMR measurements, samples of IR820, PEG-diamine, and IRPDcov were dissolved in deuterated methanol. The ${ }^{1} \mathrm{H}$ NMR spectra were recorded on a $400 \mathrm{MHz}$ Avance Bruker NMR spectrometer (Bruker Corp., Billerica, MA, USA), using 240 scans. Acquired data was processed and analyzed using MestReNova software. Chemical shifts were reported in parts per million (ppm) for ${ }^{1} \mathrm{H}$ NMR on a $\delta$ scale based on the deuterated methanol solvent peak $(\delta=3.31 \mathrm{ppm})$ as an internal standard.

\section{Dye content determination}

The IR820 content in the freeze-dried nanoconjugates was determined by dissolving a known amount of sample in DMSO, sonicating to ensure complete dissolution, and performing serial dilutions in the fluorescence linear range. Sample fluorescence was measured at peak emission after $785 \mathrm{~nm}$ excitation, with a FluoroLog ${ }^{\circledR}-3$ spectrofluorometer (Horiba Jobin Yvon, Kyoto, Japan), under constant operating conditions. The amount of IR820 in the samples was determined from a calibration curve of free IR820 in DMSO after $785 \mathrm{~nm}$ excitation, using blank subtraction.

\section{Characterization of absorption properties}

We prepared samples of IR820, PEG-diamine, and IRPDcov in deionized water at $100 \mu \mathrm{g} / \mathrm{mL}$ concentration and performed serial dilutions in the linear range. The concentration of IRPDcov was normalized to dye content. Sample absorption, from 200 to $900 \mathrm{~nm}$, was measured with a Cary UV Spectrophotometer (Agilent Technologies, Santa Clara, CA, USA).

\section{Fluorescence spectra and fluorescence stability studies}

We prepared samples of IR820 and IRPDcov in phosphatebuffered saline (PBS) buffer, at concentrations in the linear range, and measured their baseline fluorescence emission profile after $785 \mathrm{~nm}$ excitation. Sample emission fluorescence was measured from $795 \mathrm{~nm}$ up to $850 \mathrm{~nm}$, at $1 \mathrm{~nm}$ intervals, using the FluoroLog-3 spectrofluorometer. Different aliquots from the same sample batch were kept in the refrigerator and measured, following the same procedures, for up to 72 hours after preparation. The emission peak value was used to determine changes from baseline, and the percent remaining fluorescence intensity was calculated. Statistical significance was determined by comparing percent remaining intensities, using a $t$-test $(\alpha=0.05)$.

\section{Cytotoxicity with and without hyperthermia}

The cytotoxicity of four different treatments (IR820, IRPDcov, IR820 plus laser, and IRPDcov plus laser) was studied in cancer cell lines MES-SA, Dx5, and SKOV-3, using the sulforhodamine B (SRB) colorimetric assay. Cells were seeded onto 96-well plates at an approximate density of $6.2 \times 10^{4}$ cells $/ \mathrm{cm}^{2}$ and incubated overnight for attachment to the well, followed by treatment with free IR820 or IRPDcov. The SRB assay was performed 24 hours posttreatment to determine net cell growth. Tested IR820 concentrations ranged from 0 to $5 \mu \mathrm{M}$, where IR820 concentration equal to zero meant that only DPBS and no drug was added to the wells (control group). Tested IRPDcov concentrations were normalized to IR820 content in the particles so that the treatments were equivalent to tested concentrations of free dye.

In order to test the effect of hyperthermia, some of the cells were incubated for 1 hour with one of the following treatments: $5 \mu \mathrm{M}$ IR820, IRPDcov at concentrations equivalent to $5 \mu \mathrm{M}$ of IR-820, or only cell medium. These cells were then exposed to $808 \mathrm{~nm}$ laser illumination for 3 minutes, and the SRB assay was performed 24 hours postlaser treatment. Temperature was measured with a thermocouple, and experimental wells were separated from each other by at least one empty well in all directions to avoid cross-effects. Net growth values were calculated by normalizing the data from each treatment to the growth of the control cells, which 
were not exposed to IR820, IRPDcov, or laser. Statistical significance $(P<0.05)$ was identified by one-way analysis of variance (ANOVA) for the difference among groups at the same treatment concentration, and between each group and the control group.

\section{Cellular imaging}

Lysine-coated coverslips were placed in each well of a 24-well plate. MES-SA, Dx5, and SKOV-3 cells were seeded on the coverslips at a density of $5.2 \times 10^{4}$ cells $/ \mathrm{cm}^{2}, 5.2 \times 10^{4} \mathrm{cells} / \mathrm{cm}^{2}$, and $2.6 \times 10^{4}$ cells $/ \mathrm{cm}^{2}$, respectively, in McCoy's $5 \mathrm{~A}$ medium supplemented with $10 \%$ fetal bovine serum (FBS) and $1 \%$ penicillin, and were grown overnight for attachment. The next day, the cell medium was removed and replaced with $5 \mu \mathrm{M}$ IR820 or equivalent free-dye content of IRPDcov. Cells were incubated in the dark at $37^{\circ} \mathrm{C}$ for 4 hours and then washed three times with Dulbecco's phosphate-buffered saline (DPBS). Then the cells were fixed with $4 \%$ formaldehyde for 15 minutes at $37^{\circ} \mathrm{C}$, followed by washing three times with DPBS. The coverslips were then removed and mounted on glass microslides with antifade reagent/mounting medium mixture. The cells were then examined by fluorescence microscopy (Olympus IX81; Olympus Corporation, Tokyo, Japan) with a $60 \times$ water-merged objective. The fluorescence was imaged at $\lambda_{\mathrm{ex}}(775 \mathrm{~nm}), \lambda_{\mathrm{em}}(845 \mathrm{~nm})$, with an exposure time of 4,000 ms, which is well below the $20,000 \mathrm{~ms}$ threshold for autofluorescence for this wavelength. A charge-coupled device (CCD) camera was used to capture the signals, and the images were software-merged with pseudo color. The fluorescence microscope settings were kept the same throughout the experiment. The acquired fluorescence images were processed by MATLAB ${ }^{\circledR}$ (The MathWorks Inc., Natick, MA, USA) to calculate the image ratio (R). First, the intensity of each pixel was background-subtracted, and a region of interest was defined as being composed of any pixels with above-background intensity values (defined as an intensity of at least two out of a 255 scale after background subtraction). The ratio $\mathrm{R}$ was then determined by normalizing the total pixel intensity of this region of interest to its total area.

\section{Animal study design}

All animal protocols were approved by the Florida International University Institutional Animal Care and Use Committee. Thirty-six healthy ND4 Swiss Webster outbred mice, weighing between 25 and 30 grams, were purchased from Harlan Laboratories (Indianapolis, IN, USA), housed under a 12-hour light/dark cycle and fed ad libitum. Mice were randomly assigned to one of twelve groups $(n=3)$ based on optical agent (ICG, IR820, or IRPDcov) and time point of data collection after injection (15 minutes, 30 minutes, 60 minutes, and 24 hours). Solutions for injection were prepared based on a $0.24 \mathrm{mg} / \mathrm{kg}$ dose of free dye, ${ }^{28}$ which in the case of IRPDcov, was based on conjugate dye content.

For time groups in the range of minutes, mice were injected with intraperitoneal (IP) pentobarbital $(40 \mathrm{mg} / \mathrm{kg}$ for initial anesthesia during imaging, and an additional $60 \mathrm{mg} / \mathrm{kg}$ for euthanasia after imaging was completed). The animals were shaved, placed supine on the imaging setup described in the "In vivo imaging techniques" section below, injected through the tail vein with their assigned agent, and imaged. At the predetermined time point, a blood sample was obtained, under anesthesia, by heart puncture into the ventricle, and the animal was then euthanized by removal of the heart.

For mice in the 24-hour groups, each animal was anesthetized with $40 \mathrm{mg} / \mathrm{kg}$ of IP pentobarbital, injected with the assigned agent, and imaged. These mice were then returned to their cage alive until the 24 -hour time point. After 24 hours, they were again anesthetized with pentobarbital, and imaging was performed under the same operating conditions. After imaging, a blood sample was obtained by heart puncture while under anesthesia, and the animals were euthanized by removal of the heart. Organs were carefully harvested for imaging and subsequent homogenization and dye extraction in DMSO.

\section{In vivo imaging techniques}

An imaging system consisting of a Sanyo DL 7140-201S laser ( $80 \mathrm{~mW}, 785 \mathrm{~nm}$ ) and a Retiga 1300 CCD camera was used. The entire setup was covered by BK5 blackout material. The power at the imaging plane ranged from $0.1-0.5 \mathrm{~mW}$. An image of the mouse was obtained under white light illumination to determine the position of the target and to focus the camera before dye injection. Then, the laser was turned on, and another image was taken prior to dye injection, to establish background. Laser current was set to $60 \mathrm{~mA}$. After the white and background images were obtained, the dye solution was injected through the tail vein. The camera started recording immediately before the injection, and 10-second exposure images of the abdomen were recorded in series, using QCapture Pro software, for at least 10 minutes. The same procedures were followed for 24-hour imaging, except that a still picture was taken rather than serial images.

\section{Plasma and organ sample processing}

Plasma and organ sample processing followed the procedures described by other researchers who have performed 
biodistribution studies of NIR dyes and related compounds. ${ }^{29,30}$ In the case of blood samples, immediately after collection, the samples were centrifuged two times for 3 minutes at $12,000 \mathrm{rpm}$, to extract plasma. Plasma samples were then incubated in DMSO (1:50 [plasma:DMSO]) in glass vials covered with aluminum foil for 30 minutes and centrifuged again. The resulting supernatant was used to perform spectrofluorometric measurements of dye concentration in plasma, using a previously created calibration curve of IR820 or ICG in DMSO at $785 \mathrm{~nm}$ excitation.

For organ samples in the 24-hour groups, organs were carefully dissected and placed in black-coated Petri dishes for imaging. Fluorescent organs were then cut into small pieces using a scalpel, placed in preweighed glass vials, and homogenized. Then, $5 \mathrm{~mL}$ of DMSO was added for dye extraction. Samples were incubated in DMSO for 4 hours and centrifuged at 9,000 rpm for 15 minutes. The supernatant was used to perform spectrofluorometric measurements of dye content, using a previously created calibration curve of IR820 or ICG in DMSO at $785 \mathrm{~nm}$ excitation. To report the data, organ dye content was normalized to organ mass.

\section{Pharmacokinetic analysis of plasma data}

Dye concentration in plasma obtained from spectrofluorometer measurements was averaged for each group, and the average concentration for each time point was entered into MATLAB. The initial concentration (concentration at injection) for an average $25 \mathrm{~g}$ mouse was estimated to be approximately $4 \mu \mathrm{g}$ dye $/ \mathrm{mL}$ in plasma. Using the Curve Fitting Tool in MATLAB, average data points at 0, 15, 30, 60 minutes, and 24 hours were fit to biexponential curves via a least squares algorithm to represent a two-compartment

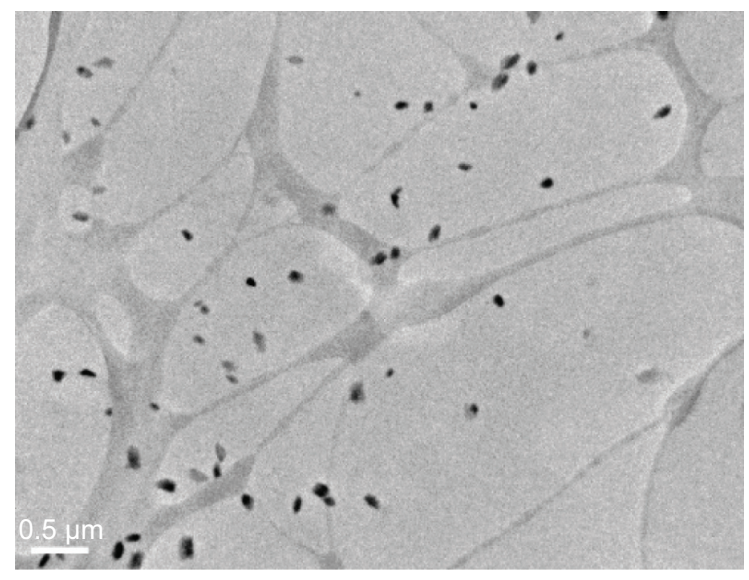

model. The resulting biexponential fit equations were used to determine the half-lives of distribution and elimination, the area under the pharmacokinetic curve (AUC), the mean plasma residence time, and the clearance rate. The mean residence time in plasma, $t_{p}$, is the average time spent by the agent of interest in plasma, and is given, in its simplest expression, by:

$$
\begin{aligned}
& \text { Mean plasma } \\
& \text { residence time }
\end{aligned}=t_{p}=\frac{\text { Area under curve }}{\text { Initial concentration }}
$$

The total body clearance rate (ie, volume of agent cleared from the body per unit time) is given by:

$$
\text { Total body clearance rate }=\frac{\text { Dose }}{\text { Area under curve }}
$$

Based on the preceding calculations, we compared the IRPDcov, IR820, and ICG pharmacokinetic parameters, using one-way ANOVA $(P<0.05)$ followed by Bonferroni post hoc test.

\section{Results and discussion Characterization of IRPDcov}

IRPDcov size and morphology were characterized using TEM, which showed nearly spherical, uniformly distributed nanoconjugates of approximately $150 \mathrm{~nm}$ diameter (Figure 2). The zeta potential of IRPDcov was $-0.4 \pm 0.3 \mathrm{mV}$ due to charge neutralization during formulation. The nanoconjugates are soluble in aqueous solution, and PEGmediated steric repulsion assists in preventing the formation of aggregates.

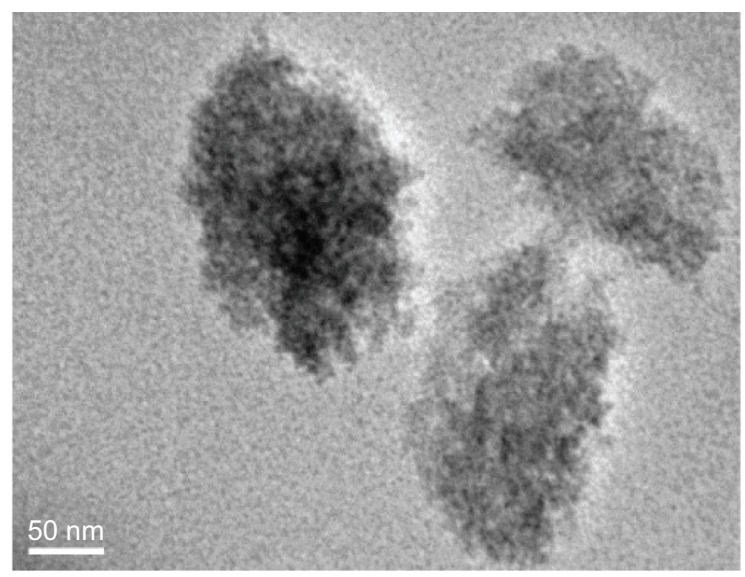

Figure 2 TEM images of IRPDcov.

Abbreviations: IRPDcov, covalent conjugates of IR820 and PEG-diamine; PEG, polyethylene glycol; TEM, transmission electron microscopy. 
Particle yield was $74 \%$ for a $6 \mathrm{kDa}$ PEG-diamine formulation. Samples of IRPDcov in deuterated methanol were also analyzed by ${ }^{1} \mathrm{H}$ NMR, which confirmed conjugate formation. The conjugate ${ }^{1} \mathrm{H}$ NMR spectrum contained peaks contributed both from IR820 and PEG-diamine, which suggested that we had indeed prepared the conjugate. Any unreacted IR820 would have been removed during the dialysis process, so the presence of aromatic peaks (in the region between 7 and 9 ppm) indicated that both IR820 and PEG-diamine were present in the conjugated structure. Additionally, the spectrum of the conjugate showed an upfield proton shift in the 7.5-8.5 ppm area compared with the spectrum of free IR820. This is likely explained by the fact that conjugation replaces the electron-withdrawing chlorine group in IR820 with an electron-releasing amine group from PEG-diamine, and indicates that the covalent linkage between IR820 and PEG-diamine was successful. IRPDcov fluorescent dye content was determined by spectrofluorometry to be approximately $0.113 \mathrm{mg}$ IR $820 / \mathrm{mg}$ IRPDcov.

\section{Absorbance studies}

The ultraviolet (UV)-visible spectra of IR820, PEG-diamine, and IRPDcov are shown in Figure 3. Concentrations of
PEG-diamine and IR820 were chosen based on molar ratios used in the formulation of the nanocomplexes, and IRPDcov concentration was chosen to approximately match the concentration of the IR820 solution, given the previously estimated dye content ( $0.113 \mathrm{mg}$ IR820/mg IRPDcov). IRPDcov displayed a bathochromic main absorption peak shift compared with IR820 (IRPDcov $\lambda_{\text {max }}=702 \mathrm{~nm}, \operatorname{IR} 820 \lambda_{\text {max }}=688 \mathrm{~nm}$ ) as well as a shift in the secondary absorption peak (located at $836 \mathrm{~nm}$ for IRPDcov versus $815 \mathrm{~nm}$ for IR820). These shifts can be attributed to the formation of an extended $\pi$ system by the conjugation process. There was also an overall broadening of the spectral profile. Increased absorption and scattering within the environment of the nanoconjugate may explain the spectral observations. There were no peaks above 0.01 AU between 200 and $900 \mathrm{~nm}$ for PEG-diamine at $24 \mu \mathrm{g} / \mathrm{mL}$. The free-dye spectrum showed a maximum peak at $688 \mathrm{~nm}$, with almost the same absorption intensity as the IRPDcov solution, confirming our dye content determination.

\section{Fluorescence properties of IRPDcov and fluorescence stability studies}

A representative fluorescence emission spectrum of IRPDcov after $785 \mathrm{~nm}$ excitation is shown in Figure 4, along with the

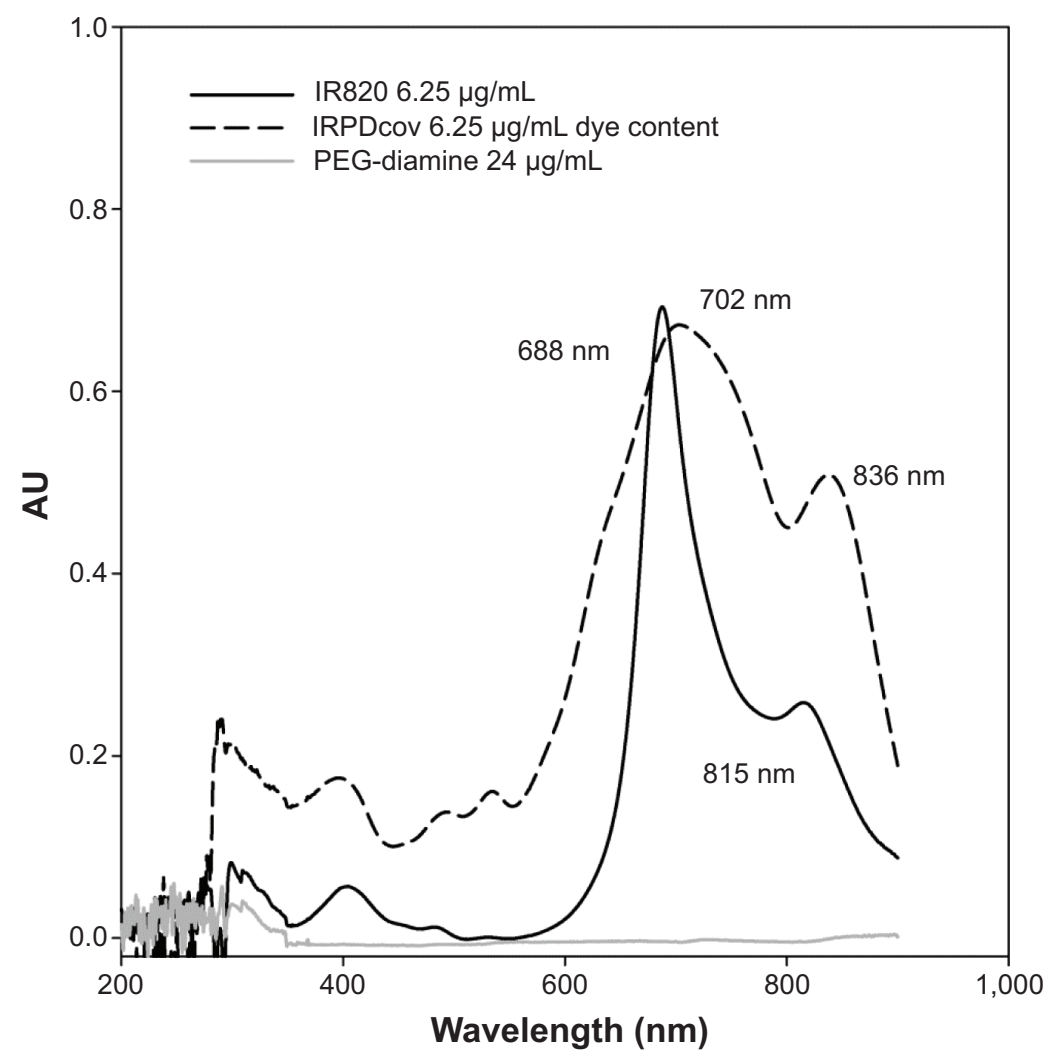

Figure 3 Absorption spectra of IR820 (solid black), IRPDcov (dashed black), and PEG-diamine (gray) in deionized water. Abbreviations: IRPDcov, covalent conjugates of IR820 and PEG-diamine; PEG, polyethylene glycol; AU, absorbance units. 


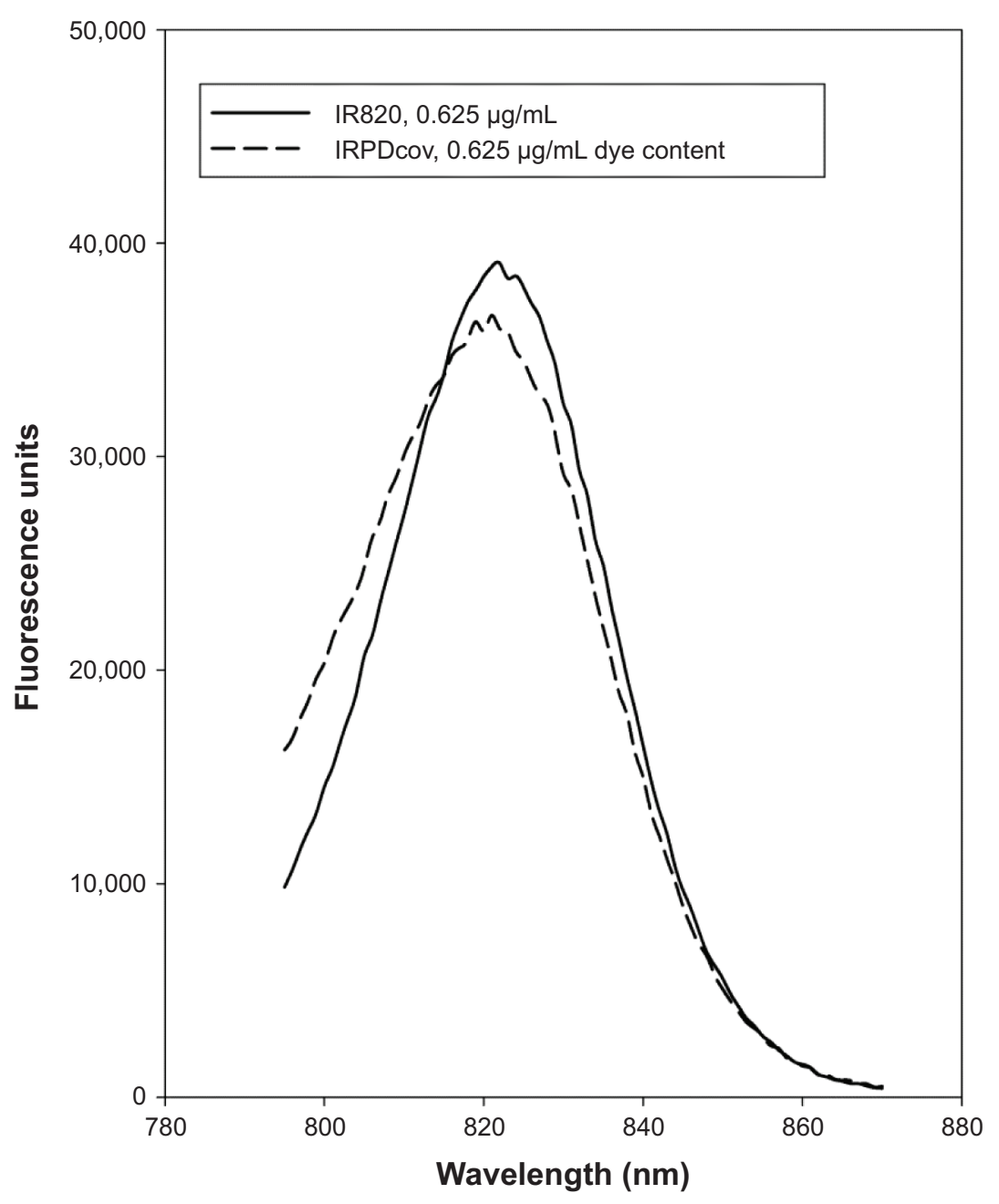

Figure 4 Fluorescence spectra of IR820 and IRPDcov in phosphate-buffered saline.

Abbreviations: IRPDcov, covalent conjugates of IR820 and PEG-diamine; PEG, polyethylene glycol.

corresponding profile for IR820 at comparable dye content. After $785 \mathrm{~nm}$ excitation, the peak emission for IRPDcov was located at $822 \mathrm{~nm}$, with fluorescence intensity 6\% smaller than IR820. The slight reduction in fluorescence intensity may have been due to increased scattering within the structure or to secondary self-absorption due to the presence of the $836 \mathrm{~nm}$ absorption peak. IRPDcov and IR820 solutions in buffer were measured up to 72 hours after preparation, to determine the percent remaining fluorescent intensity compared with baseline. Samples were kept at $4{ }^{\circ} \mathrm{C}$, in the dark. The decrease in fluorescence from baseline after 72 hours was significantly lower for IRPDcov (39.9\% decrease) compared with IR820 $(80 \%$ decrease $)(P<0.05)$. This indicates that covalent conjugation provides significant stabilization to the dye, likely as a result of increased rigidity of the IR820 molecule from its incorporation into the nanoconjugate lattice, which would reduce intermolecular IR820 aggregation. PEG-mediated steric hindrance may also have played a role in preventing aggregation of IR820 molecules and the subsequent degradation process. The enhanced preservation of fluorescence properties also indicates that the conjugate was stable as formulated for at least 72 hours, based on the large differences in fluorescence observed between the conjugate and free-dye samples after that time period.

\section{Cellular imaging}

Figure 5 shows fluorescence microscopy images of Dx5, SKOV-3, and MES-SA cell lines after 4 hours incubation at $5 \mu \mathrm{M}$ concentration of IR820 and IRPDcov at equivalent dye content. This concentration was chosen based on our previous cellular studies with IR820. In all three cell lines, intense fluorescence was observed inside the cells after 4-hour incubation with IR820 or IRPDcov, indicating that both the free dye and the nanoconjugates were capable of entering the cells. However, the images taken after IRPDcov incubation showed higher normalized intensity ratios than 
A

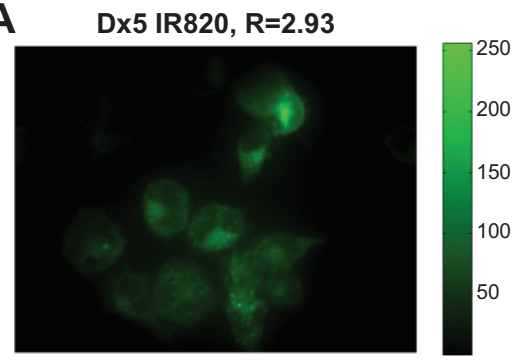

B
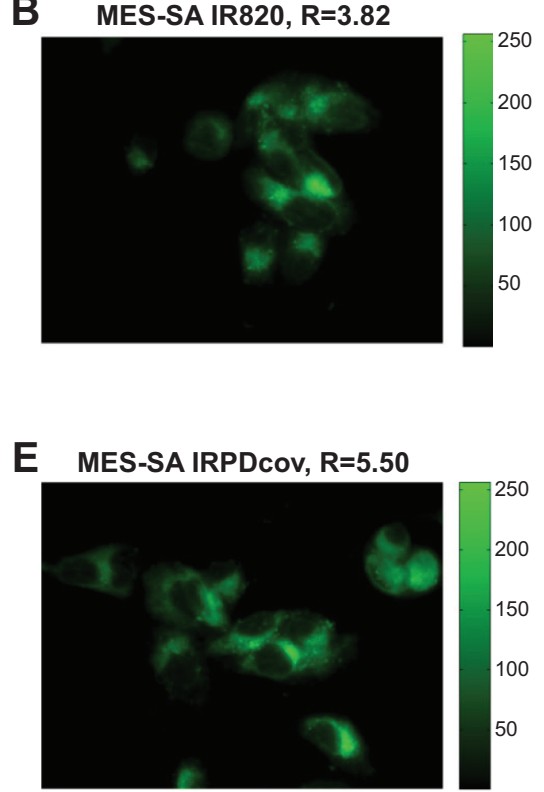

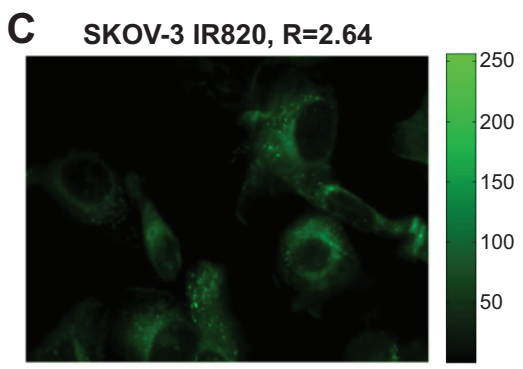

F SKOV-3 IRPDcov, $\mathrm{R}=3.21$

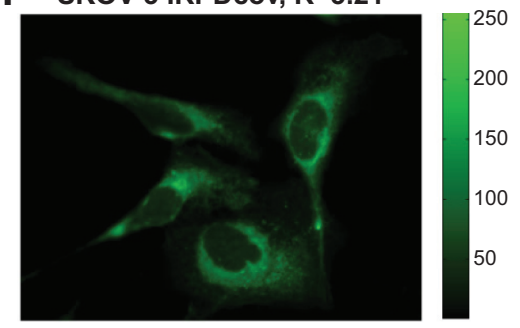

Figure 5 Cellular imaging of Dx5 (A and D), MES-SA (B and E), and SKOV-3 cells (C and F) after a 4-hour incubation with $5 \mu$ M dye content of IR820 (top) or IRPDcov (bottom); 60×, exposure time 4,000 ms, compared against the pixel intensities scaled from 0 to 255 .

Abbreviations: IRPDcov, covalent conjugates of IR820 and PEG-diamine; PEG, polyethylene glycol.

did those taken after incubation with equivalent concentrations of IR820, especially in Dx5. Normalized intensity ratios were 1.8 times larger for IRPDcov than for IR820 in Dx5, 1.4 times larger in MES-SA, and 1.2 times larger in SKOV-3. PEG can enhance cell membrane interaction and increase cell internalization, by osmoelastic coupling and formation of PEG-induced fusion vesicles. ${ }^{31,32}$ In the case of IRPDcov, this may be one of the contributing factors to enhanced internalization.

\section{Cytotoxicity and hyperthermia studies}

When excited with $808 \mathrm{~nm}$ light, IR820 spontaneously releases a significant amount of energy in the form of heat. In response to photon excitation, electrons in the IR820 molecule transition to different energy levels, and the subsequent process of relaxation results in heat dissipation. We tested IRPDcov to determine whether the dye in nanoconjugate form was still able to generate heat upon exposure to an $808 \mathrm{~nm}$ NIR laser. At a laser fluence rate of $8 \mathrm{~W} / \mathrm{cm}^{2}$, a solution of $0.038 \mathrm{mg} / \mathrm{mL}$ IRPDcov (containing $5 \mu \mathrm{M}$ IR820) caused an increase in temperature from $37^{\circ} \mathrm{C}$ to $42.2^{\circ} \mathrm{C}$ after 3 minutes of exposure, demonstrating that covalent binding of IR820 did not interfere with its heat generation properties. This temperature is in the moderate hyperthermia range $\left(41^{\circ} \mathrm{C}-43^{\circ} \mathrm{C}\right)$, which can cause significant tumor cell growth inhibition but does not greatly affect healthy cells. It has been reported that cancer cells have mechanisms that inhibit oxidative metabolism that are specifically activated by temperatures in this range. ${ }^{33-36} \mathrm{We}$ also tested PEG-diamine by itself, which did not result in significant changes in temperature after 3-minute laser exposure compared with wells containing medium only. Therefore, the IR820 component of the nanoconjugates was responsible for hyperthermia generation.

SKOV-3, Dx5, and MES-SA cells were chosen to study the cytotoxic effect of IR820 and IRPDcov on tumor cells, with and without hyperthermia. Figure 6 shows net cell growth following incubation with IR820 or IRPDcov, with or without exposure to laser, for SKOV-3, Dx5, and MES-SA. All groups were normalized to the control group not exposed to dye or laser. From our previous studies, we determined that laser exposure by itself or PEG-diamine by itself did not cause any significant effect on cell growth for any of the three cell lines. ${ }^{21}$

Without laser, IRPDcov demonstrates toxicity comparable to the free dye. There was no significant toxicity in SKOV-3 or Dx5 compared with the control group, which was not exposed to IRPDcov or IR820, and there was a slight growth inhibition in MES-SA cells for either treatment, consistent with our previous reports for IR820 and IR820 conjugates. ${ }^{11,21}$ There was no significant difference in net cell growth between the IR820 group and the IRPDcov group without laser exposure for any cell line, indicating that IRPDcov can be safely used up to at least $5 \mu \mathrm{M}$. 


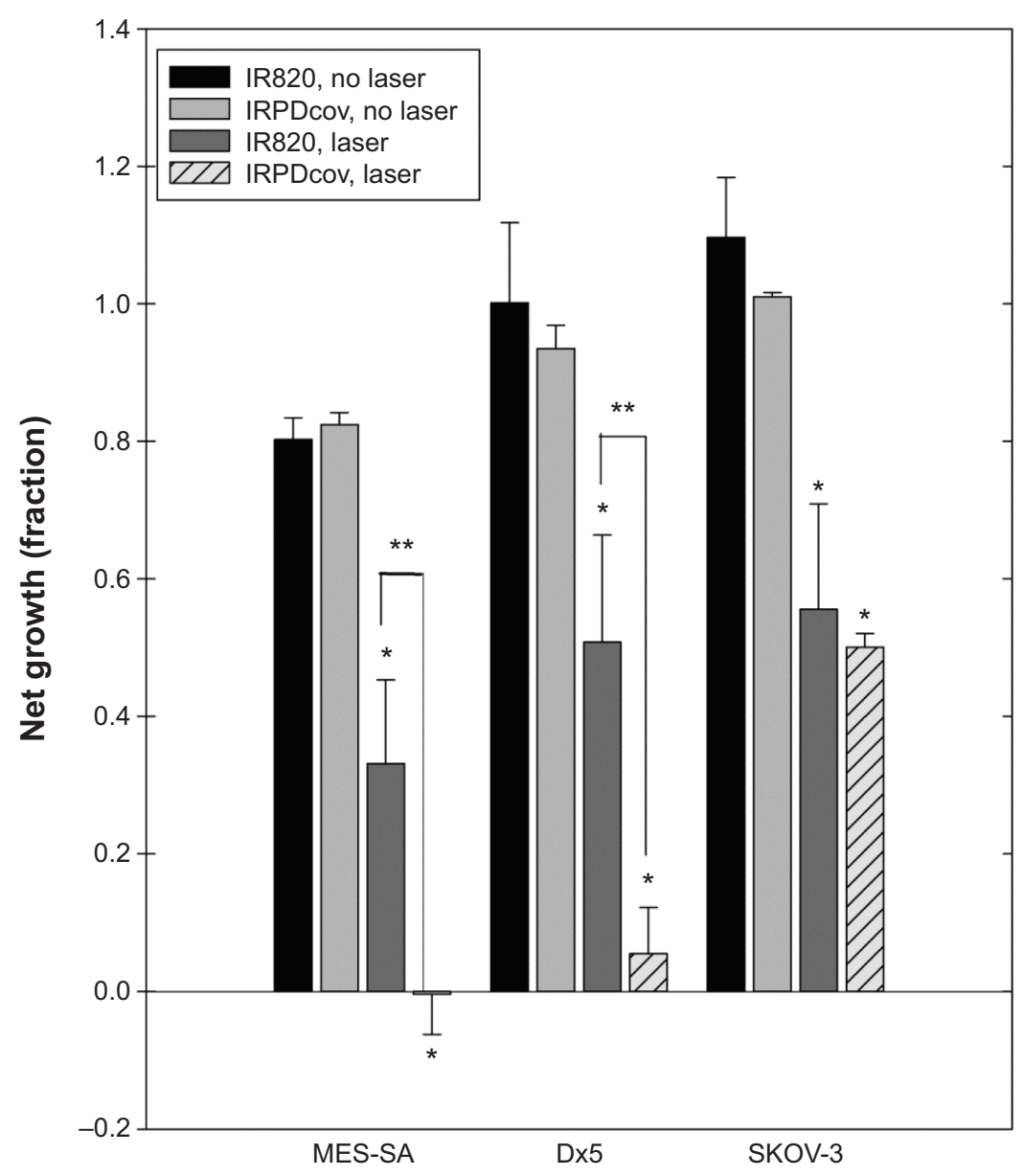

Figure 6 Cytotoxicity of IRPDcov or IR820 in cancer cell lines, with or without hyperthermia treatment at $5 \mu$ M dye concentration, $\mathrm{n}=3$ experiments, 4 wells/experiment. Notes: All groups are normalized to the control group not exposed to dye or laser. There was no significant effect of laser exposure per se without IRPDcov or IR820, and there was no significant effect of PEG-diamine with or without exposure to laser. *Significant difference $(P<0.05)$ versus nonlaser group; $* *$ significant difference $(P<0.05)$ between laser groups.

Abbreviations: IRPDcov, covalent conjugates of IR820 and PEG-diamine; PEG, polyethylene glycol.

When exposed to laser, cell growth was significantly inhibited in all three cell lines by both IR820 and IRPDcov. In MES-SA and Dx 5 cell lines, exposure to IRPDcov with hyperthermia resulted in significantly higher cytotoxicity $(P<0.05)$ compared with IR820. In the case of MES-SA, IRPDcov plus laser resulted in cell killing, whereas IR820 plus laser caused only cell growth inhibition. In SKOV-3 cells, IRPDcov with hyperthermia produced higher cytotoxicity than did free dye but not enough to reach statistical significance. Based on the cell imaging results displayed in Figure 5, there may have been a larger degree of internalization for IRPDcov than for free dye. Although this is not a direct measurement of uptake, the results of the image analysis seem to indirectly indicate that there may have been higher amounts of conjugate than free dye present in the cells. This would be consistent with the increased cytotoxicity effect of the hyperthermia treatment that can be observed in Figure 6.
Enhanced internalization would result in larger accumulation of the dye inside the cell and an accentuated damaging effect of temperature increases. This seems to be most apparent in MES-SA cells, where treatment with IRPDcov plus laser resulted in a cell-killing effect.

It is interesting to note that the hyperthermia-enhanced cytotoxic effect versus free dye reached statistical significance in MES-SA and Dx5 cell lines but not in SKOV-3. Other researchers have reported that SKOV-3 cells have inherent thermotolerance, which could be related to their characteristic $p 53$ gene mutation. ${ }^{37}$ Hyperthermiainduced cytotoxicity is controlled by both $p 53$-dependent and -independent pathways. ${ }^{38-40}$ Based on existing knowledge, thermosensitivity and thermotolerance depend on an array of biological, genetic, and environmental factors so that a specific cause for SKOV-3 thermotolerance within the context of our studies cannot be determined. 


\section{Animal and organ imaging}

Figure 7 shows representative images obtained from ICG, IR820, and IRPDcov animals 15 minutes and 24 hours after injection. These images prove that IRPDcov can be used for in vivo imaging and that it provides an imaging signal comparable to that of IR820. The signal ratio for ICG at 15 minutes after injection was higher than that of IR820 or IRPDcov; however, by 24 hours, the intensity of ICG had dropped significantly in comparison with the other two agents.

The 24-hour ICG signal also seemed to be located in the lower abdomen, instead of in the liver region as is the case for the other two agents. Organ images obtained after 24 hours demonstrate a very different biodistribution for ICG compared with the other two agents. Figure 8 provides a qualitative comparison of organ signals for IRPDcov, IR820, and ICG, and Table 1 summarizes the organ signal intensity per unit area ratios for each dye.

The information obtained from the ratio data indicates that the biodistribution of IRPDcov, IR820, and ICG shows a different pattern between agents 24 hours after intravenous (IV) injection. Although an ICG signal was still present in the liver, the ratio was significantly lower $(P<0.05)$ than for IR820 or IRPDcov. The amount of ICG present in the kidneys and the lungs was also significantly smaller $(P<0.05)$ than for the other two agents. In the case of the intestines, a higher signal was observed for ICG than for IR820 or IRPDcov; however, the effect did not reach statistical significance, due to the large standard deviation for the ICG measurements in the intestines. We expect that intersubject variability in intestinal motility rates would affect ICG more markedly, given that most of the dye is being eliminated via the gastrointestinal tract at the 24-hour time point.

In the case of IR820, a strong signal was observed in the liver, kidneys, and lungs, with a small signal in the intestines. IRPDcov showed a strong signal in the liver, some signal in the kidneys, and a small signal in lungs and intestines. Statistical analysis comparing IR820 and IRPDcov showed that there were no differences in liver or intestinal image ratios. There was a significant difference $(P<0.05)$ in lung image ratios, with IRPDcov showing a smaller signal ratio than IR820. This possibly indicates that the nanoformulation was able to escape detection by alveolar macrophages, thanks to the presence of PEG, which reduces binding to serum proteins. ${ }^{41}$ In the kidneys, the IRPDcov signal was smaller than that of IR820, but the difference did not reach statistical significance. There may be a somewhat smaller degree of elimination of IRPDcov through the kidneys, which would be consistent with the expectation that the larger size of the conjugate compared with the free dye would result in decreased renal clearance (based on a low molecular size cutoff for renal excretion), ${ }^{42}$ as well as the fact that the presence of PEG could result in reduced reticuloendothelial system uptake. However, the small number of subjects in our study may not provide enough sensitivity to detect a significant difference. Another reason for the lack of significance could
A

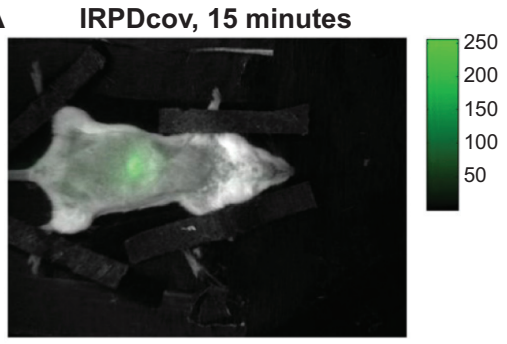

D

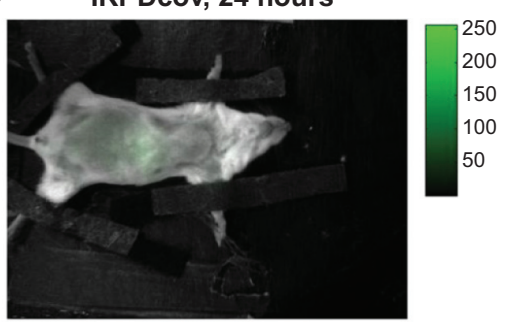

B

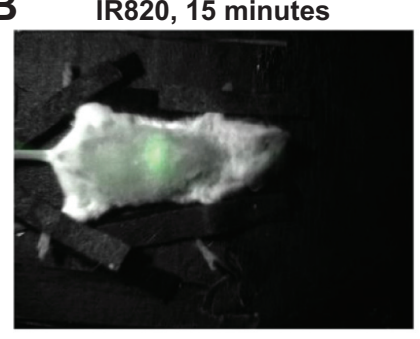

E

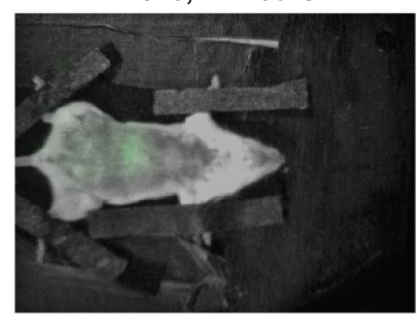

C
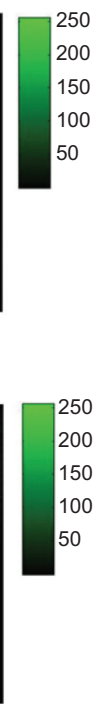

$\mathbf{F}$
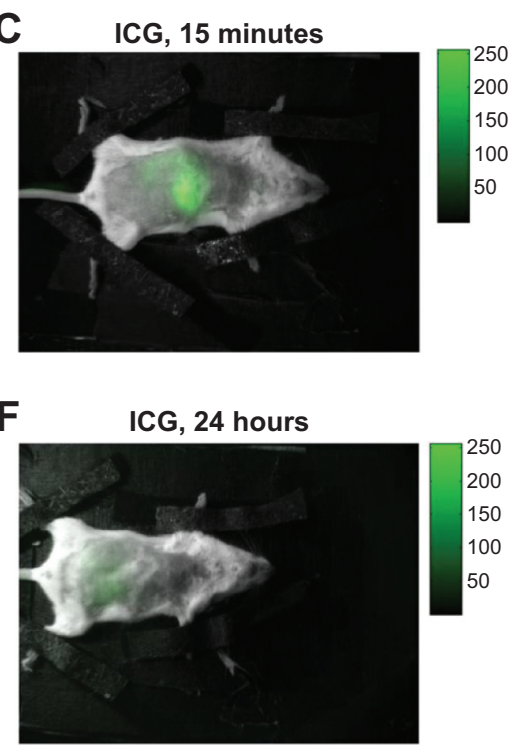

Figure 7 Images taken 15 minutes and 24 hours after IV injection for IRPDcov (A and D), IR820 (B and E), and ICG (C and F) compared against the pixel intensities scaled from 0 to 255 .

Note: Mice were shaved for imaging.

Abbreviations: ICG, indocyanine green; IRPDcov, covalent conjugates of IR820 and PEG-diamine; IV, intravenous; PEG, polyethylene glycol. 

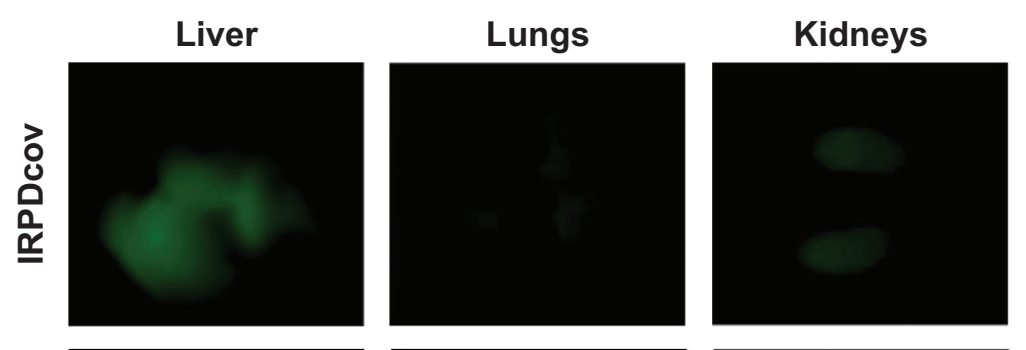

\section{Intestines}
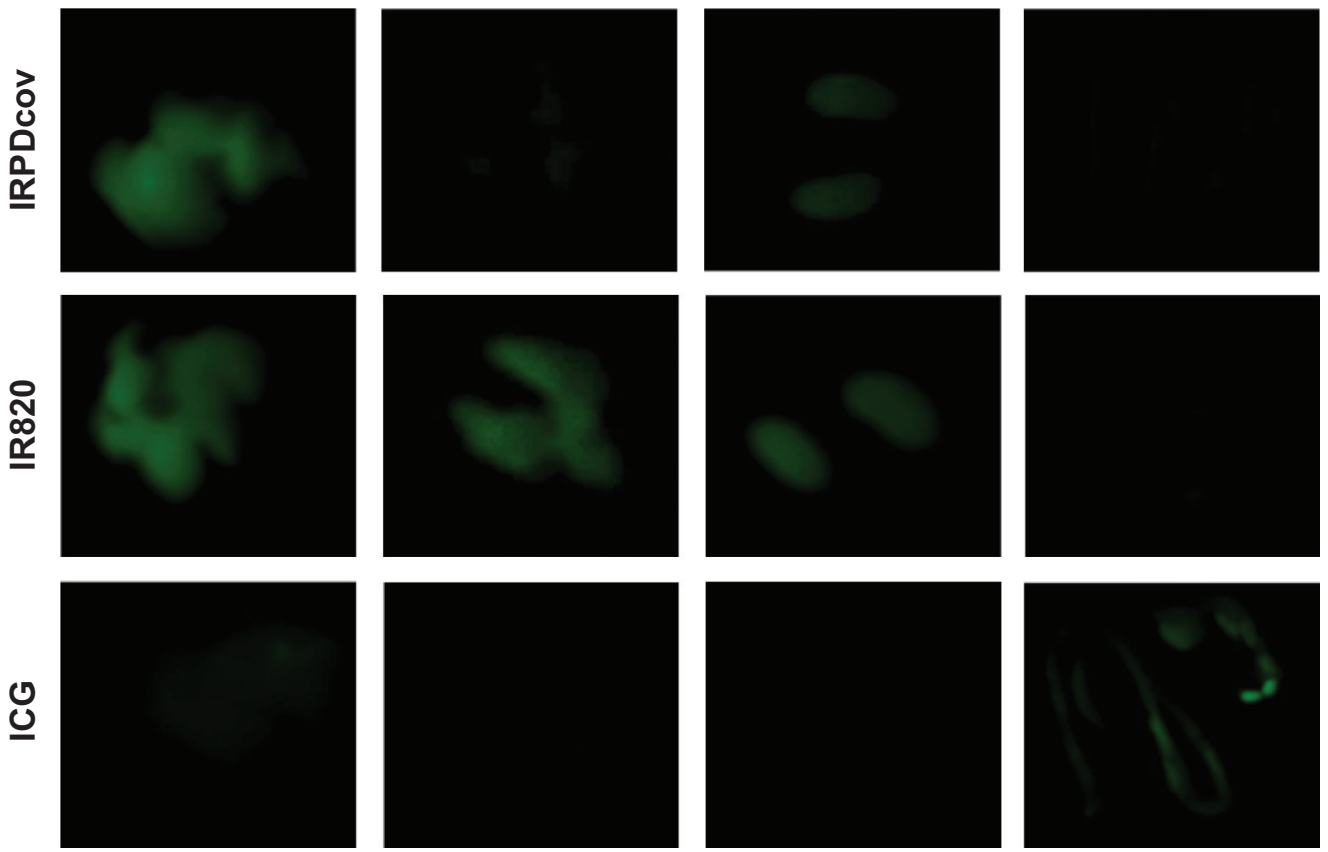

Figure 8 Organ images taken 24 hours after IV injection of IRPDcov, IR820, or ICG compared against the pixel intensities scaled from 0 to 255 . Abbreviations: ICG, indocyanine green; IRPDcov, covalent conjugates of IR820 and PEG-diamine; IV, intravenous; PEG, polyethylene glycol.

be variability in renal elimination rates between subjects. Additionally, imaging ratios may not be sensitive enough to detect quantitative differences.

From these images, we can conclude that there are clear differences in the biodistribution patterns of ICG versus IRPDcov and IR820, with ICG being more rapidly cleared from major organs than the other two agents, and primarily localizing in fecal elimination pathways by 24 hours. The difference in behavior was probably due to a much faster plasma clearance combined with a high level of ICG binding to plasma proteins, faster liver clearance, and loss of signal due to poor ICG stability. IR820 and IRPDcov provide an advantage in terms of prolonged residence

Table I Signal intensity ratios for liver, lungs, kidneys and intestines collected 24 hours after IV injection of IRPDcov, IR820, or ICG

\begin{tabular}{llll}
\hline & \multicolumn{3}{l}{ Image intensity per area, $\mathbf{R}\left(\mathrm{I} / \mu^{2}\right)$} \\
\cline { 2 - 4 } & IRPDcov & IR820 & ICG \\
\hline Liver & $6.35 \pm 0.44^{*}$ & $6.13 \pm 1.44^{*}$ & $0.76 \pm 0.35$ \\
Lungs** & $0.64 \pm 0.08^{*}$ & $2.48 \pm 0.47^{*}$ & $0.18 \pm 0.32$ \\
Kidneys & $1.34 \pm 0.13^{*}$ & $2.95 \pm 1.88^{*}$ & $0.20 \pm 0.34$ \\
Intestines & $0.65 \pm 0.10$ & $1.00 \pm 0.45$ & $2.01 \pm 1.43$ \\
\hline
\end{tabular}

Notes: Values represent average \pm SD. *Significant difference $(P<0.05)$ with ICG values for the same organ; **significant difference $(P<0.05)$ between IRPDcov and IR820 values for the same organ.

Abbreviations: ICG, indocyanine green; IRPDcov, covalent conjugates of IR820 and PEG-diamine; IV, intravenous; PEG, polyethylene glycol; SD, standard deviation. times, although for both these agents, the timing of imaging would need to be optimized so that the signal is high in the target tissue and low in the plasma and nontarget tissue. In terms of comparing IRPDcov with IR820, our lung images support the hypothesis of reduced mononuclear phagocyte system uptake, ${ }^{43}$ which we can probably owe to the presence of PEG. However, this is not the only possible factor affecting the biodistribution of these conjugates as size can also play a significant role in the pharmacokinetics of nanoformulations and their uptake by body tissues. For instance, particles of sizes larger than $10 \mathrm{~nm}$ are unlikely to undergo glomerular filtration, and the primary elimination route for particles that are not excreted through the kidneys is the hepatobiliary system, ${ }^{44}$ consistent with our observations.

\section{Organ dye content}

Figure 9 shows the average dye content (in $\mu \mathrm{g}$ dye/g tissue) for liver, lungs, kidneys, and intestines 24 hours after an IV injection of IRPDcov, IR820, or ICG. The data is consistent with our qualitative observations and quantitative signal ratios from the optical imaging samples, and provides increased sensitivity to detect differences. When we compare the results for ICG with those of IRPDcov and IR820, we see that organ content was significantly higher in the intestines and significantly lower in all other organs. For lungs and 


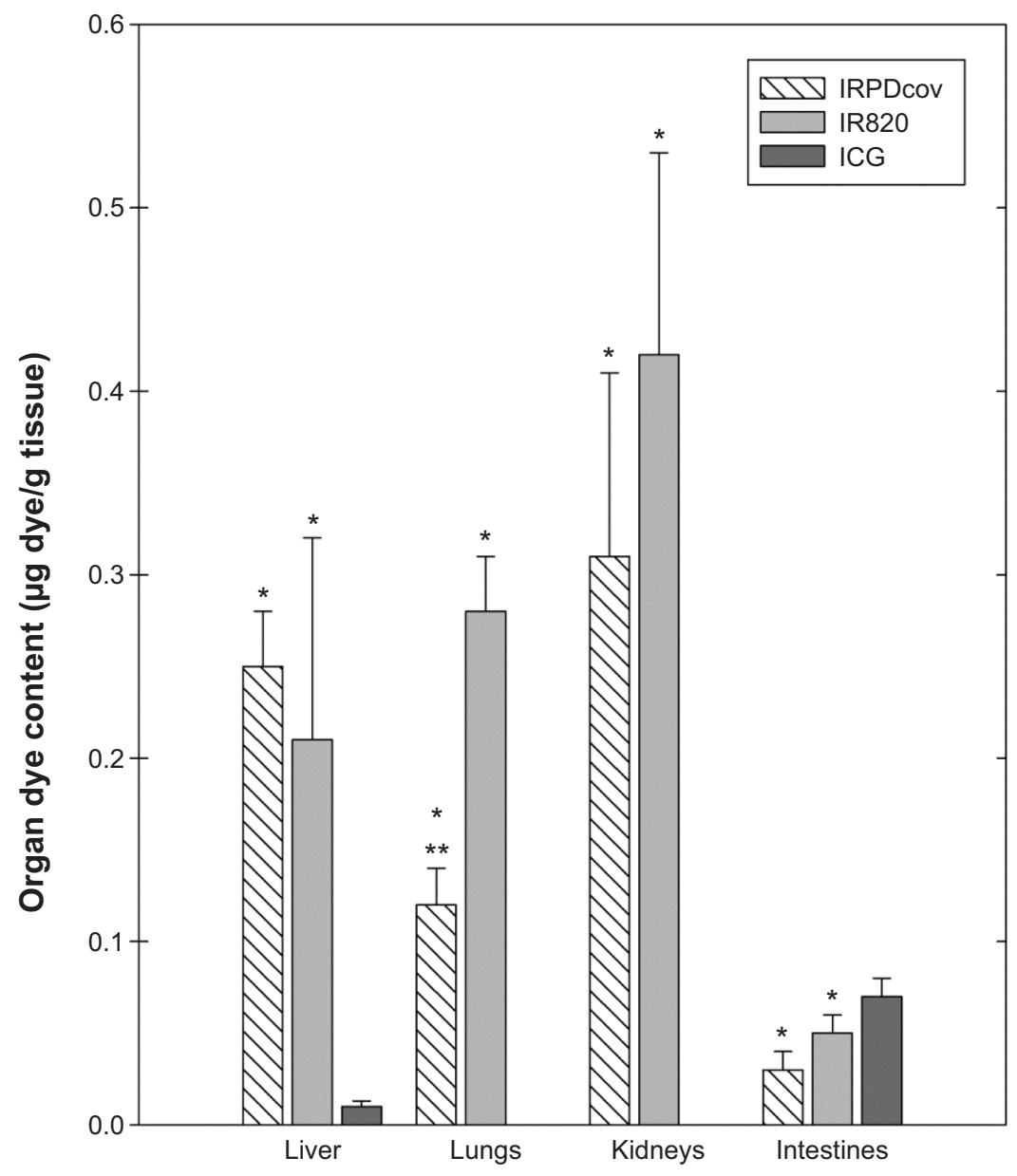

Figure 9 Quantitative organ content 24 hours after IV injection of IRPDcov, IR820, or ICG.

Notes: *Significant difference $(P<0.05)$ with ICG values for same organ; **significant difference $(P<0.05)$ between IRPDcov and IR820 values for same organ. There was no detectable signal for ICG in lungs or kidneys after 24 hours.

Abbreviations: ICG, indocyanine green; IRPDcov, covalent conjugates of IR820 and PEG-diamine; IV, intravenous; PEG, polyethylene glycol.

kidneys, ICG sample readings were at background level. When comparing IRPDcov and IR820, the only significant difference was a reduced content of IRPDcov in the lungs. IRPDcov kidney content was lower than that for IR820, but the difference was not significant. This is consistent with our observations in the organ imaging studies. The difference between intestinal uptake of ICG, IRPDcov, and IR820 became significant once quantitative organ dye content was used as a measurement.

\section{Plasma dye concentration}

Figure 10 shows the average dye concentration in plasma (in $\mu \mathrm{g} / \mathrm{mL}$ ) for IRPDcov, IR820, and ICG at each of the time points under study. We observed significantly higher $(P<0.05)$ plasma concentrations of IR820 and IRPDcov compared with ICG for all time points, with plasma values 20 times higher for IRPDcov and 10 times higher for IR820, at 24 hours. We also found significantly higher $(P<0.05)$ concentrations of IRPDcov compared with IR820 at the 30-minute, 60-minute, and 24-hour time points, with plasma concentration of IRPDcov double that of IR820 at the 24-hour sampling time.

Based on these results, not only were IR 820 formulations superior to ICG in terms of plasma concentration over time, but we also observed a clear advantage of the IRPDcov conjugate over IR820 after initial distribution. This validates the theoretical basis for the formulation of this conjugate, which relied on the possibility that the presence of PEG could contribute to improved plasma concentration profiles by reducing detection by macrophages and other circulating agents in plasma. IRPDcov is thus analogous to other formulations in which the inclusion of PEG has provided some stealth characteristics in an in vivo environment. ${ }^{45-49}$

The recovered percent age of injected dose at 24 hours in plasma was $3.3 \%$ for IRPDcov, $1.7 \%$ for IR 820 , and $0.8 \%$ 


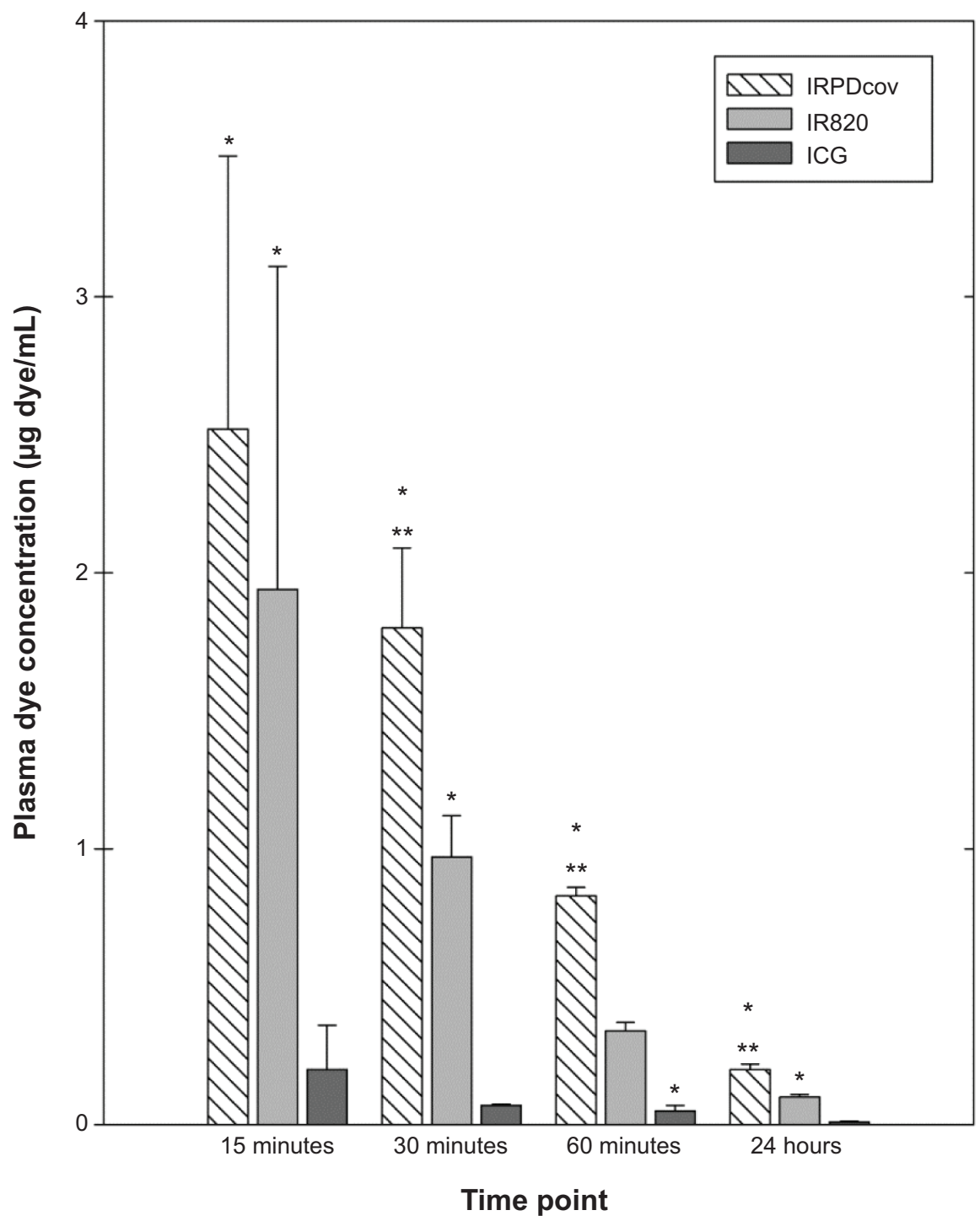

Figure 10 Plasma concentrations $(\mu \mathrm{g} / \mathrm{mL})$ of IRPDcov, IR820, and ICG at different time points after IV injection. Notes: *Significant difference from ICG $(P<0.05)$; **significant difference IRPDcov vs IR820 $(P<0.05)$.

Abbreviations: ICG, indocyanine green; IRPDcov, covalent conjugates of IR820 and PEG-diamine; IV, intravenous; PEG, polyethylene glycol.

for ICG. If we add these numbers to the percentage values of the recovered injected doses found in our organ content studies, we reach an overall percentage of $15.1 \%$ for IRPDcov, $17.7 \%$ for IR 820 , and $2.6 \%$ for ICG. In the case of ICG, almost the entire injected dose was lost after 24 hours, which demonstrates a clear advantage of the IR820 formulations in terms of injected dose recovery at this time point. Surprisingly, we did not observe a larger percentage dose recovery for IRPDcov compared with IR820. A possible explanation may be that the circulating concentration of IRPDcov was still double that of IR820 at 24 hours. It is also possible that a considerable amount of the conjugate may yet have been present in other nonsampled tissues, such as fat, skin, muscle, bone, or the lymphatic system.

\section{Pharmacokinetic modeling}

Table 2 shows the resulting fitted parameters from a twocompartment pharmacokinetics model of the IRPDcov, IR820, and ICG data, given the biexponential equation:

$$
C(t)=A \times e^{-a t}+B \times e^{-b t}
$$

Our results show that both IR820 and IRPDcov demonstrated significantly longer $(P<0.05)$ distribution half-lives, longer elimination half-lives, larger AUC, longer plasma residence times, and slower body clearance rates than did ICG. Although distribution happened quite fast for all agents, the ICG distribution half-life was approximately 3.06 minutes, whereas the value for IR820 was 13.4 minutes and for 
Table 2 Pharmacokinetic parameters from two-compartment analysis of dye data in mice

\begin{tabular}{|c|c|c|c|}
\hline & IRPDcov & IR820 & ICG \\
\hline $\begin{array}{l}\text { Model equation based } \\
\text { on plasma data }\end{array}$ & $C(t)=3.66 \times \mathrm{e}^{-1.91 t}+0.32 \times \mathrm{e}^{-0.020 t}$ & $C(t)=3.83 \times e^{-3.12 t}+0.1702 \times e^{-0.023 t}$ & $C(t)=3.93 \times e^{-13.54 t}+0.07 \times e^{-0.38 t}$ \\
\hline$R^{2}$ & 0.995 & 0.999 & 0.999 \\
\hline Distribution half-life, $h$ & $0.364 \pm 0.006^{t, t \dagger}$ & $0.223 \pm 0.003^{t, t \dagger}$ & $0.05 I \pm 0.001$ \\
\hline Elimination half-life, $h$ & $35.46 \pm 0.56^{t, t \dagger}$ & $30.45 \pm 0.45^{t, t \dagger}$ & $1.85 \pm 0.03$ \\
\hline AUC, $(\mu g-h) / m L$ & $7.98 \pm 0.13^{t, t \dagger}$ & $4.38 \pm 0.07^{t,+\dagger}$ & $0.490 \pm 0.007$ \\
\hline Mean residence time in plasma, $\mathrm{h}$ & $1.99 \pm 0.03^{t+t \dagger}$ & $1.09 \pm 0.02^{\dagger, t \dagger}$ & $0.12 \pm 0.002$ \\
\hline Total body clearance rate, $\mathrm{mL} / \mathrm{h}$ & $0.75 \pm 0.0 I^{t, t+}$ & $1.37 \pm 0.02^{\dagger+t \dagger}$ & $12.25 \pm 0.18$ \\
\hline
\end{tabular}

Notes: †Significant difference $(P<0.05)$ with ICG; +†significant difference $(P<0.05)$ IRPDcov vs IR820.

Abbreviations: AUC, area under the time-concentration curve; ICG, indocyanine green; IRPDcov, covalent conjugates of IR820 and PEG-diamine; PEG, polyethylene glycol.

IRPDcov was 21.8 minutes. The distribution half-life value for ICG is consistent with that previously reported by other authors, which has ranged from 2 to 4 minutes. ${ }^{50,51}$ The calculated elimination half-lives for IRPDcov and IR820, which both exceeded 30 hours, were in stark contrast with that of ICG, which was 1.85 hours. These values are also well aligned with some of the conclusions reached by Prajapati et al who estimated that IR 820 should have a clearance half-life close to that of albumin, which is in the order of 35 hours. ${ }^{14}$ The elimination half-life value we obtained for ICG is consistent with literature data as other authors report an elimination half-life of over 1 hour at low concentrations. ${ }^{50}$

Both the distribution and the elimination phases showed improved profiles for IR820 formulations compared with ICG, in terms of providing a wider time window for theranostic action. As far as the AUC, both IRPDcov and IR820 were an order of magnitude larger than ICG, indicating an increased overall tissue exposure to the theranostic agent. Additionally, IRPDcov also showed a significant advantage $(P<0.05)$ over IR820 for all the above calculated parameters, with almost double the AUC and mean plasma residence time of IR820, and almost half the clearance rate of IR820. The nanoformulation was present in plasma for longer periods of time, eliminated more slowly from the body, and the cumulative exposure of body compartments to the conjugate was larger than for either of the two free dyes. The time that the IRPDcov was bioavailable was also significantly longer as the elimination half-life of IRPDcov was 1.16 times longer than that of IR820 and 19 times longer than that of ICG, whereas the distribution half-life of IRPDcov was 1.6 times longer than that of IR820 and seven times longer than that of ICG.

Given that the enhanced permeability and retention effect is proportional to the time and amount of agent circulating in blood, ${ }^{29}$ we would theoretically expect IRPDcov to result in a greater amount of dye accumulated and retained in tumors.
However, further studies in a tumor-bearing model would be needed to explore this possibility.

Other authors have studied the pharmacokinetics of nanoformulations, and the general consensus is that nanoformulations may result in improved pharmacokinetic profiles, in many cases as a result of stabilization of the drug and reduced metabolism and clearance. ${ }^{42}$ Still, loss of nanocomplexes from the circulation can occur by binding to serum proteins in the process known as surface opsonization, which subsequently causes recognition and removal by circulating phagocytes or macrophages. ${ }^{47,52}$ In the case of PEGylated formulations, steric hindrance reduces the degree of protein binding, resulting in prolonged circulation times and giving PEGylated formulations an improved pharmacokinetic profile compared with their non-PEGylated counterparts. ${ }^{42,52}$ This seems to be applicable to formulations incorporating PEG as a conjugated moiety, as was the case of the IRPDcov conjugate.

Another consideration which is intimately tied to these profiles is the surface charge of the formulation. Our nanoconjugates were zwitterionic, presenting both positive and negative charges and an overall zeta potential approaching neutrality. Other authors have found that for other physical characteristics being similar, neutral and zwitterionic nanoformulations exhibit prolonged plasma half-lives and reduced clearance compared with largely positive or largely negative formulations, which indicates that charge may play a large role in maximizing circulation time. ${ }^{53}$

Nanocomplex size also has an effect on circulation time, with particles around $100 \mathrm{~nm}$ diameter demonstrating longer blood circulation profiles than larger or smaller particle sizes. $^{42}$ Liu et al studied the effect of size on biodistribution of liposomes and found that sizes between 100 and $200 \mathrm{~nm}$ were present in circulation for longer times than those greater than $250 \mathrm{~nm}$ or smaller than $50 \mathrm{~nm} .{ }^{54}$ Given that our nanoconjugates were around $150 \mathrm{~nm}$ per TEM measurement, 
it seems that size was also contributing to their prolonged circulation times.

Our analysis is not without possible limitations. First, the pharmacokinetic models we used have important assumptions, such as uniform distribution, no absorption, rapid equilibration before sampling, and no degradation. Uniform distribution may be an issue as we know that nanoformulations are distributed to tissues depending on pore size, in a different manner than their free-form counterparts. ${ }^{55,56}$ In terms of rapid equilibration, others have reported that equilibration of injected solutions in mice plasma can be assumed to happen within 2 minutes. ${ }^{57}$ Since our first sample was taken at 15 minutes, we can assume that equilibration had occurred by that time. Regarding the possibility of degradation into other byproducts, other authors have shown that ICG is mostly excreted in its intact form in bile. ${ }^{29}$ Our in vitro studies show that IRPDcov conjugates were very stable and retained their characteristics for at least 72 hours in PBS. Based on this, we do not expect degradation to be a significant concern within the time periods studied in vivo. However, the in vivo environment cannot be fully mimicked by in vitro conditions, and some in vivo factors, such as the presence of plasma proteins, reticuloendothelial system scavengers, and metabolism in liver or other tissues, may result in aggregation, degradation, or changes in the structure of these molecules.

Image-guided therapy using IR820 conjugates shows promise for clinical translation as it can be coupled with minimally invasive light delivery techniques, such as endoscopic or orthoscopic approaches. Future work will focus on studying the biodistribution of these conjugates in tumor-bearing animals in order to determine in vivo tumor uptake, as well as on the design and optimization of minimally invasive in vivo methods for combined imaging and hyperthermia, possibly through fiber optic technology.

\section{Conclusion}

We successfully prepared nanocomplexes of IR820 and PEG-diamine (IRPDcov) and investigated cellular uptake and cytotoxicity in cancer cell lines MES-SA, SKOV-3, and Dx5. The IRPDcov formulation can potentially provide an advantage over the use of free agents by significantly enhancing the stability of the NIR dye, increasing cell internalization, allowing simultaneous colocalization of imaging and therapy, and accentuating the cytotoxic effect of hyperthermia. IRPDcov can be used for in vitro optical imaging of cancer cells as well as in vivo imaging. Biodistribution and pharmacokinetic studies of IRPDcov, IR820, and ICG in healthy mice show that IRPDcov is a feasible alternative to IR820 for in vivo imaging and that it demonstrates an improved pharmacokinetic profile over the free-dye form as well as over the commonly used NIR dye ICG. IRPDcov demonstrated significantly longer distribution and elimination half-live, longer mean plasma residence time, larger overall exposure as indicated by AUC, and slower clearance rate compared with either IR820 or ICG. The conjugate has different organ localization than free IR820, with potential reduced accumulation in the kidneys and significantly lower $(P<0.05)$ accumulation in the lungs. The nanosize nature of our conjugate, as well as surface and charge characteristics provided by the presence of PEG, is likely to be responsible for these differences. The use of IRPDcov conjugates could provide wider in vivo availability windows for theranostic applications. Future research will include studies in tumorbearing animals, in order to explore the passive targeting ability of this formulation as well as to investigate the optimal dosage that will provide effective therapeutic hyperthermia and imaging in vivo.

\section{Acknowledgments}

This work was funded in part by National Institutes of Health (NIH) grant number 1R15CA167571-01A1. The authors would like to acknowledge Dr Pradeep Kumar for his advice regarding formulation of the conjugate, Dr Joong-Ho Moon and Ms Tereza Vokata for their help in acquisition of ${ }^{1} \mathrm{H}$ NMR spectra, and Ms Dorybel Irias for assisting with conjugate preparation and characterization. TEM was done at the Advanced Materials Engineering Research Institute (AMERI). The experiments described in this manuscript were completed as partial fulfillment of the requirements for Alicia Fernandez-Fernandez' PhD dissertation. Portions of this work have been included in her dissertation manuscript, ${ }^{58}$ as well as in conference abstracts for the 2013 SPIE-Bios Conference (San Francisco, CA). ${ }^{59}$

\section{Disclosure}

The authors report no conflicts of interest in this work.

\section{References}

1. Xie J, Lee S, Chen X. Nanoparticle-based theranostic agents. Adv Drug Deliv Rev. 2010;62(11):1064-1079.

2. Fernandez-Fernandez A, Manchanda R, McGoron AJ. Theranostic applications of nanomaterials in cancer: drug delivery, image-guided therapy, and multifunctional platforms. Appl Biochem Biotechnol. 2011;165(7-8):1628-1651.

3. Janib SM, Moses AS, MacKay JA. Imaging and drug delivery using theranostic nanoparticles. Adv Drug Deliv Rev. 2010;62(11):1052-1063.

4. Lu ZR. Theranostics: fusion of therapeutics and diagnostics. Pharm Res. 2014;31(6):1355-1357. 
5. Wang D, Lin B, Ai H. Theranostic nanoparticles for cancer and cardiovascular applications. Pharm Res. 2014;31(6):1390-1406.

6. Ryu JH, Lee S, Son S, et al. Theranostic nanoparticles for future personalized medicine. J Control Release. 2014;190:477-484.

7. Kelkar SS, Reineke TM. Theranostics: combining imaging and therapy. Bioconjug Chem. 2011;22(10):1879-1903.

8. Sumer B, Gao J. Theranostic nanomedicine for cancer. Nanomedicine (Lond). 2008;3(2):137-140.

9. Melancon MP, Stafford RJ, Li C. Challenges to effective cancer nanotheranostics. J Control Release. 2012;164(2):177-182.

10. Frangioni JV. In vivo near-infrared fluorescence imaging. Curr Opin Chem Biol. 2003;7(5):626-634.

11. Fernandez-Fernandez A, Manchanda R, Lei T, et al. Comparative study of the optical and heat generation properties of IR 820 and indocyanine green. Mol Imaging. 2012;11(2):99-113.

12. Tang Y, Lei T, Manchanda R, et al. Simultaneous delivery of chemotherapeutic and thermal-optical agents to cancer cells by a polymeric (PLGA) nanocarrier: an in vitro study. Pharm Res. 2010;27(10): 2242-2253.

13. Srinivasan S, Manchanda R, Lei T, Nagesetti A, Fernandez-Fernandez A, McGoron AJ. Targeted nanoparticles for simultaneous delivery of chemotherapeutic and hyperthermia agents - an in vitro study.J Photochem Photobiol B. 2014;136:81-90.

14. Prajapati SI, Martinez CO, Bahadur AN, et al. Near-infrared imaging of injured tissue in living subjects using IR-820. Mol Imaging. 2009; 8(1):45-54.

15. Pandey RK, James N, Chen Y, Dobhal MP. Cyanine-based compounds for tumor imaging with and without photodynamic therapy. Top Heterocycl Chem. 2008;14:41-74.

16. Masotti A, Vicennati P, Boschi F, Calderan L, Sbarbati A, Ortaggi G A novel near-infrared indocyanine dye-polyethylenimine conjugate allows DNA delivery imaging in vivo. Bioconjug Chem. 2008;19(5): 983-987.

17. Thierry B, Al-Ejeh F, Khatri A, et al. Multifunctional core-shell magnetic cisplatin nanocarriers. Chem Commun (Camb). 2009;(47): 7348-7350.

18. Srinivasan S, Manchanda R, Fernandez-Fernandez A, Lei T, McGoron AJ. Near-infrared fluorescing IR820-chitosan conjugate for multifunctional cancer theranostic applications. $J$ Photochem Photobiol B. 2013;119:52-59.

19. Lei T, Fernandez-Fernandez A, Manchanda R, Huang YC, McGoron AJ. Near-infrared dye loaded polymeric nanoparticles for cancer imaging and therapy and cellular response after laser-induced heating. Beilstein J Nanotechnol. 2014;5:313-322.

20. Lei T, Manchanda R, Fernandez-Fernandez A, Huang YC, Wright $\mathrm{D}$, McGoron AJ. Thermal and $\mathrm{pH}$ sensitive multifunctional polymer nanoparticles for cancer imaging and therapy. RSC Adv. 2014; 4(34):17959-17968.

21. Manchanda R, Fernandez-Fernandez A, Carvajal DA, Lei T, Tang Y, McGoron AJ. Nanoplexes for cell imaging and hyperthermia: in vitro studies. J Biomed Nanotechnol. 2012;8(4):686-694.

22. Brigger I, Dubernet C, Couvreur P. Nanoparticles in cancer therapy and diagnosis. Adv Drug Delivery Rev. 2002;54(5):631-651.

23. Xu ZP, Zeng QH, Lu GQ, Yu AB. Inorganic nanoparticles as carriers for efficient cellular delivery. Chem Eng Sci. 2006;61(3):1027-1040.

24. Iyer AK, Khaled G, Fang J, Maeda H. Exploiting the enhanced permeability and retention effect for tumor targeting. Drug Discov Today. 2006;11(17-18):812-818.

25. Harris JM, Martin NE, Modi M. Pegylation: a novel process for modifying pharmacokinetics. Clin Pharmacokinet. 2001;40(7):539-551.

26. Alexis F, Pridgen E, Molnar LK, Farokhzad OC. Factors affecting the clearance and biodistribution of polymeric nanoparticles. Mol Pharm. 2008;5(4):505-515.

27. Hrkach JS, Peracchia MT, Domb A, Lotan N, Langer R. Nanotechnology for biomaterials engineering: structural characterization of amphiphilic polymeric nanoparticles by $1 \mathrm{H}$ NMR spectroscopy. Biomaterials. 1997;18(1):27-30.
28. Rajagopalan R, Uetrecht P, Bugaj JE, Achilefu SA, Dorshow RB. Stabilization of the optical tracer agent indocyanine green using noncovalent interactions. Photochem Photobiol. 2000;71(3):347-350.

29. Saxena V, Sadoqi M, Shao J. Polymeric nanoparticulate delivery system for Indocyanine green: biodistribution in healthy mice. Int $J$ Pharm. 2006;308(1-2):200-204.

30. Yaseen MA, Yu J, Jung B, Wong MS, Anvari B. Biodistribution of encapsulated indocyanine green in healthy mice. Mol Pharm. 2009; 6(5):1321-1332.

31. Yamazaki M, Ito T. Deformation and instability in membrane structure of phospholipid vesicles caused by osmophobic association: mechanical stress model for the mechanism of poly(ethylene glycol)-induced membrane fusion. Biochemistry. 1990;29(5):1309-1314.

32. Zhang Y, Kohler N, Zhang M. Surface modification of superparamagnetic magnetite nanoparticles and their intracellular uptake. Biomaterials. 2002;23(7):1553-1561.

33. Cavaliere R, Ciocatto EC, Giovanella BC, et al. Selective heat sensitivity of cancer cells. Biochemical and clinical studies. Cancer. 1967;20(9): 1351-1381.

34. Luk KH, Hulse RM, Phillips TL. Hyperthermia in cancer therapy. West J Med. 1980;132(3):179-185.

35. Tang Y, McGoron AJ. Combined effects of laser-ICG photothermotherapy and doxorubicin chemotherapy on ovarian cancer cells. J Photochem Photobiol B. 2009;97(3):138-144.

36. Tang Y, McGoron AJ. Increasing the rate of heating: a potential therapeutic approach for achieving synergistic tumour killing in combined hyperthermia and chemotherapy. Int J Hyperthermia. 2013;29(2): $145-155$.

37. Van de Broek B, Devoogdt N, D'Hollander A, et al. Specific cell targeting with nanobody conjugated branched gold nanoparticles for photothermal therapy. ACS Nano. 2011;5(6):4319-4328.

38. Fukami T, Nakasu S, Baba K, Nakajima M, Matsuda M. Hyperthermia induces translocation of apoptosis-inducing factor (AIF) and apoptosis in human glioma cell lines. J Neurooncol. 2004;70(3):319-331.

39. Ohnishi T, Takahashi A, Mori E, Ohnishi K. p53 Targeting can enhance cancer therapy via radiation, heat and anti-cancer agents. Anticancer Agents Med Chem. 2008;8(5):564-570.

40. Sturm I, Rau B, Schlag PM, et al. Genetic dissection of apoptosis and cell cycle control in response of colorectal cancer treated with preoperative radiochemotherapy. BMC Cancer. 2006;6:124.

41. Jokerst JV, Lobovkina T, Zare RN, Gambhir SS. Nanoparticle PEGylation for imaging and therapy. Nanomedicine (Lond). 2011;6(4): $715-728$.

42. Li SD, Huang L. Pharmacokinetics and biodistribution of nanoparticles. Mol Pharm. 2008;5(4):496-504.

43. van Vlerken LE, Vyas TK, Amiji MM. Poly(ethylene glycol)-modified nanocarriers for tumor-targeted and intracellular delivery. Pharm Res. 2007;24(8):1405-1414.

44. Longmire M, Choyke PL, Kobayashi H. Clearance properties of nanosized particles and molecules as imaging agents: considerations and caveats. Nanomedicine (Lond). 2008;3(5):703-717.

45. Bazile D, Prud'homme C, Bassoullet MT, Marlard M, Spenlehauer G, Veillard M. Stealth Me. PEG-PLA nanoparticles avoid uptake by the mononuclear phagocytes system. J Pharm Sci. 1995;84(4):493-498.

46. Gref R, Lück M, Quellec P, et al. 'Stealth' corona-core nanoparticles surface modified by polyethylene glycol (PEG): influences of the corona (PEG chain length and surface density) and of the core composition on phagocytic uptake and plasma protein adsorption. Colloids Surf B Biointerfaces. 2000;18(3-4):301-313.

47. Moghimi SM, Hunter AC, Murray JC. Long-circulating and targetspecific nanoparticles: theory to practice. Pharmacol Rev. 2001;53(2): 283-318.

48. Peracchia MT, Harnisch S, Pinto-Alphandary H, et al. Visualization of in vitro protein-rejecting properties of PEGylated stealth polycyanoacrylate nanoparticles. Biomaterials. 1999;20(14):1269-1275.

49. Scott MD, Murad KL. Cellular camouflage: fooling the immune system with polymers. Curr Pharm Des. 1998;4(6):423-438. 
50. Desmettre T, Devoisselle JM, Mordon S. Fluorescence properties and metabolic features of indocyanine green (ICG) as related to angiography. Surv Ophthalmol. 2000;45(1):15-27.

51. Mordon S, Devoisselle JM, Soulie-Begu S, Desmettre T. Indocyanine green: physicochemical factors affecting its fluorescence in vivo. Microvasc Res. 1998;55(2):146-152.

52. Albanese A, Tang PS, Chan WC. The effect of nanoparticle size, shape, and surface chemistry on biological systems. Annu Rev Biomed Eng. 2012;14:1-16.

53. Arvizo RR, Miranda OR, Moyano DF, et al. Modulating pharmacokinetics, tumor uptake and biodistribution by engineered nanoparticles. PLoS One. 2011;6(9):e24374.

54. Liu D, Mori A, Huang L. Role of liposome size and RES blockade in controlling biodistribution and tumor uptake of GM1-containing liposomes. Biochim Biophys Acta. 1992;1104(1):95-101.

55. Cho K, Wang X, Nie S, Chen ZG, Shin DM. Therapeutic nanoparticles for drug delivery in cancer. Clin Cancer Res. 2008;14(5):1310-1316.
56. Dhanikula AB, Singh DR, Panchagnula R. In vivo pharmacokinetic and tissue distribution studies in mice of alternative formulations for local and systemic delivery of Paclitaxel: gel, film, prodrug, liposomes and micelles. Curr Drug Deliv. 2005;2(1):35-44.

57. Berezhkovskiy LM. Determination of mean residence time of drug in plasma and the influence of the initial drug elimination and distribution on the calculation of pharmacokinetic parameters. J Pharm Sci. 2009; 98(2):748-762.

58. Fernandez-Fernandez A. IR820 Nanoconjugates for Theranostic Applications [PhD Dissertation]. Miami, FL: Biomedical Engineering, Florida International University; 2013.

59. Fernandez-Fernandez A, Manchanda R, Carvajal DA, Lei T, McGoron AJ. Covalent IR820-PEG diamine conjugates: characterization and in vivo biodistribution. Paper presented at: SPIE-BIOS 2013 (San Francisco, CA). Conference Proceedings - Proc. SPIE 8596, Reporters, Markers, Dyes, Nanoparticles, and Molecular Probes for Biomedical Applications V, 859605 (February 21, 2013).
International Journal of Nanomedicine

\section{Publish your work in this journal}

The International Journal of Nanomedicine is an international, peerreviewed journal focusing on the application of nanotechnology in diagnostics, therapeutics, and drug delivery systems throughout the biomedical field. This journal is indexed on PubMed Central,

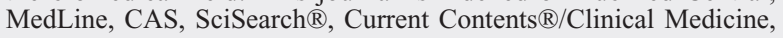

\section{Dovepress}

Journal Citation Reports/Science Edition, EMBase, Scopus and the Elsevier Bibliographic databases. The manuscript management system is completely online and includes a very quick and fair peer-review system, which is all easy to use. Visit http://www.dovepress.com/ testimonials.php to read real quotes from published authors. 